\title{
An Efficient Synthesis of $\alpha, \alpha$-Diallyl Carbinols from Esters Activated with Amides via an In-Mediated Barbier Type Allylation
}

\author{
Yu Mi Kim, Sung Hwan Kim, and Jae Nyoung Kim* \\ Department of Chemistry and Institute of Basic Science, Chonnam National University, Gwangju 500-757, Korea \\ *E-mail:kimjn@chonnam.ac.kr \\ Received March 27, 2010, Accepted April 14, 2010
}

Key Words: Indium, Barbier reaction, Amide-Esters, Diallyl carbinols

Allylindium reagents have been used extensively for the introduction of allyl group in a Barbier type manner to various electrophiles. ${ }^{1-3}$ Although many reactive electrophiles such as aldehydes and imines have been used in the indium-mediated allylations, ${ }^{1}$ the reactions of allylindium reagents with less reactive nitriles and esters have not been reported much.,3

Recently, we reported a series of indium-mediated Barbier type allylations of nitrile groups in $\gamma$-cyanoesters, ${ }^{3 a} \gamma$-ketonitriles, ${ }^{3 \mathrm{~b}} \delta$-ketonitriles, ${ }^{3 \mathrm{c}}$ and ortho-cyanobenzoates. ${ }^{3 \mathrm{~d}}$ During the studies we found that allylindium reagents, generated in situ from indium powder and allyl bromide in THF, can react with nitrile when the molecule has a suitable electrophilic quencher such as an ester, ${ }^{3 \mathrm{a}, \mathrm{d}}$ another nitrile, ${ }^{3 \mathrm{c}}$ and a sterically hindered ketone. $^{3 b, c}$ Very recently, we found that an amide group can also be used as an effective quencher for the imine intermediate to produce quinazoline derivative in good yield, as shown in Scheme 1.

During the synthesis of quinazolines we examined the reaction of allylindium reagents and oxalic amide-ester derivative 2a, prepared from 2-aminobenzonitrile (1a) and monoethyl oxalyl chloride. ${ }^{4}$ Three types of compounds could be expected as shown in Scheme 2. The first candidate is a quinazoline derivative (I) that can be formed according to the process in Scheme 1. The second possibility is the formation of a sevenmembered ring and the following second allylation to form a benzo[e]diazepine scaffold (II). The last one is a diallylation of ester moiety to produce diallyl carbinol $3 \mathbf{a}^{5}{ }^{5}$

Actually, the reaction of $\mathbf{2 a}$ and allylindium reagents, generated from allyl bromide and indium powder in THF, afforded diallyl carbinol $\mathbf{3 a}(71 \%)$ as the major product within $5 \mathrm{~min}$ at refluxing temperature. We could not observe the formation of quinazoline (I) and benzo[ $e]$ diazepine derivative (II). The results stated that the reactivity of an activated ester with amide group is larger than that of the nitrile moiety toward allylindium reagents. To the best of our knowledge the reaction of ester and allylindium reagents has not been reported, ${ }^{6}$ although the ester of $\mathbf{2} \mathbf{a}$ is an activated one with nearby amide group. Zincmediated diallylation of diethyl oxalate has been reported; ${ }^{5 \mathrm{~b}, \mathrm{c}}$ however, an In-mediated diallylation has not been reported (vide infra, Scheme 4). Encouraged by the results we decided to<smiles>C=CCc1nc(-c2ccccc2)nc2ccccc12</smiles>

Scheme 1

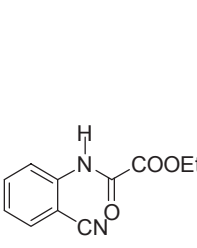

2a

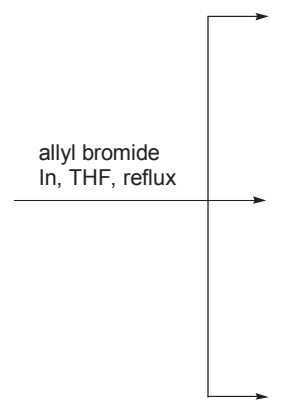

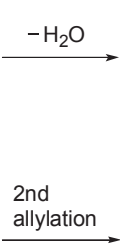

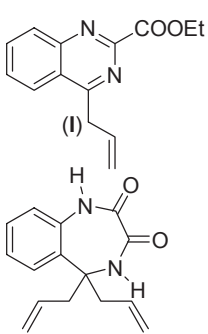

(II) 


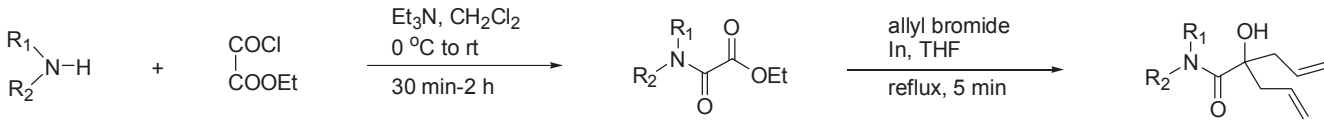

$$
\begin{aligned}
& \text { 1a-i 2a-i } \\
& 3 a-i
\end{aligned}
$$

\section{Scheme 3}
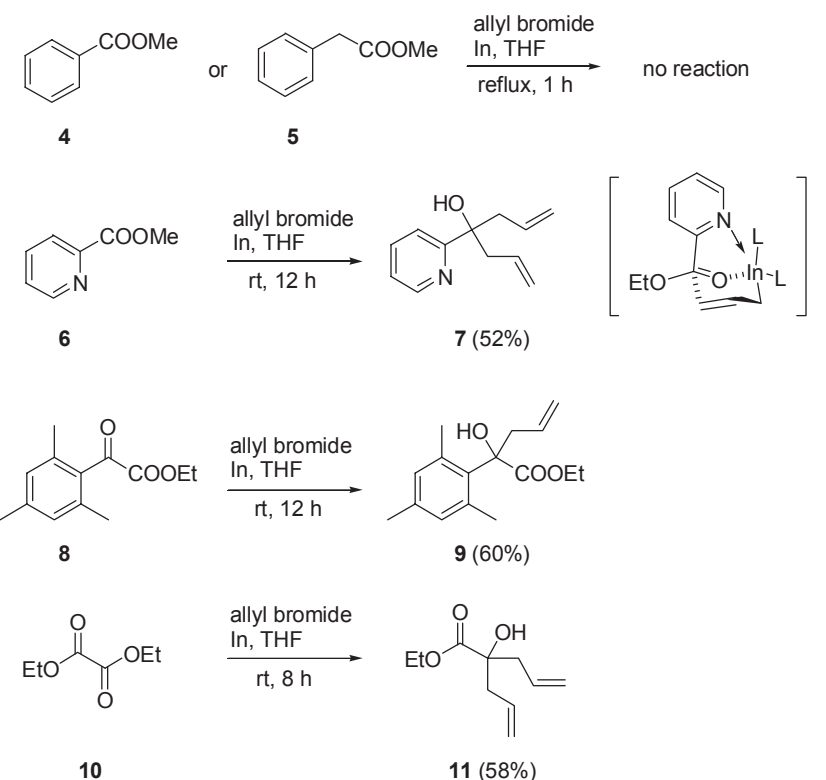

Scheme 4

examine the In-mediated allylation of oxalic amide-esters.

The starting materials 2a-i were prepared from the corresponding amines 1a-i and monoethyl oxalyl chloride $\left(\mathrm{Et}_{3} \mathrm{~N}\right.$, $\mathrm{CH}_{2} \mathrm{Cl}_{2}, 0{ }^{\circ} \mathrm{C}$ to rt, $2 \mathrm{~h}$ ) in good yields $(81-91 \%)$. ${ }^{4}$ The reaction of $\mathbf{2 b}$, as a representative ester, with allylindium reagents produced $\mathbf{3 b}$ in a reasonable yield $(75 \%)$ within 5 min at refluxing temperature. The reaction required $60 \mathrm{~min}$ for the completion at $40-45^{\circ} \mathrm{C}$ while $12 \mathrm{~h}$ at room temperature. The use of other solvents such as DMF, aqueous THF, and aqueous DMF showed similar reactivity. Thus we carried out the reactions of $\mathbf{2 c - i}$ in THF at refluxing temperature in the presence of allyl bromide (4.0 equiv) and indium powder ( 2.0 equiv), and the results are summarized in Table 1. Irrespective of the $N$-substituents the corresponding diallyl carbinols $\mathbf{3 c}$-i were obtained in good yields $(66-79 \%)$. It is interesting to note that the reaction of $2 \mathrm{e}$ (entry 5) showed the participation only at the activated ester part to produce 3e. Methallyl bromide could be used successfully in the reaction (entry 10).

As a next experiment, we examined the reactions of allylindium reagents with five representative esters, methyl benzoate (4), methyl phenylacetate (5), ethyl 2-picolinate (6), ethyl mesitylglyoxylate (8), and diethyl oxalate (10), as shown in Scheme 4. The reactions of $\mathbf{4}$ and $\mathbf{5}$ failed even at refluxing temperature. The reaction of $\mathbf{6}$ produced the corresponding diallyl carbinol 7 in moderate yield (52\%). The increased reactivity of compound $\mathbf{6}$ as compared to compound $\mathbf{4}$ must be ascribed to the pre-
Table 1. Synthesis of amide-esters 2a-i and their In-mediated allylation.

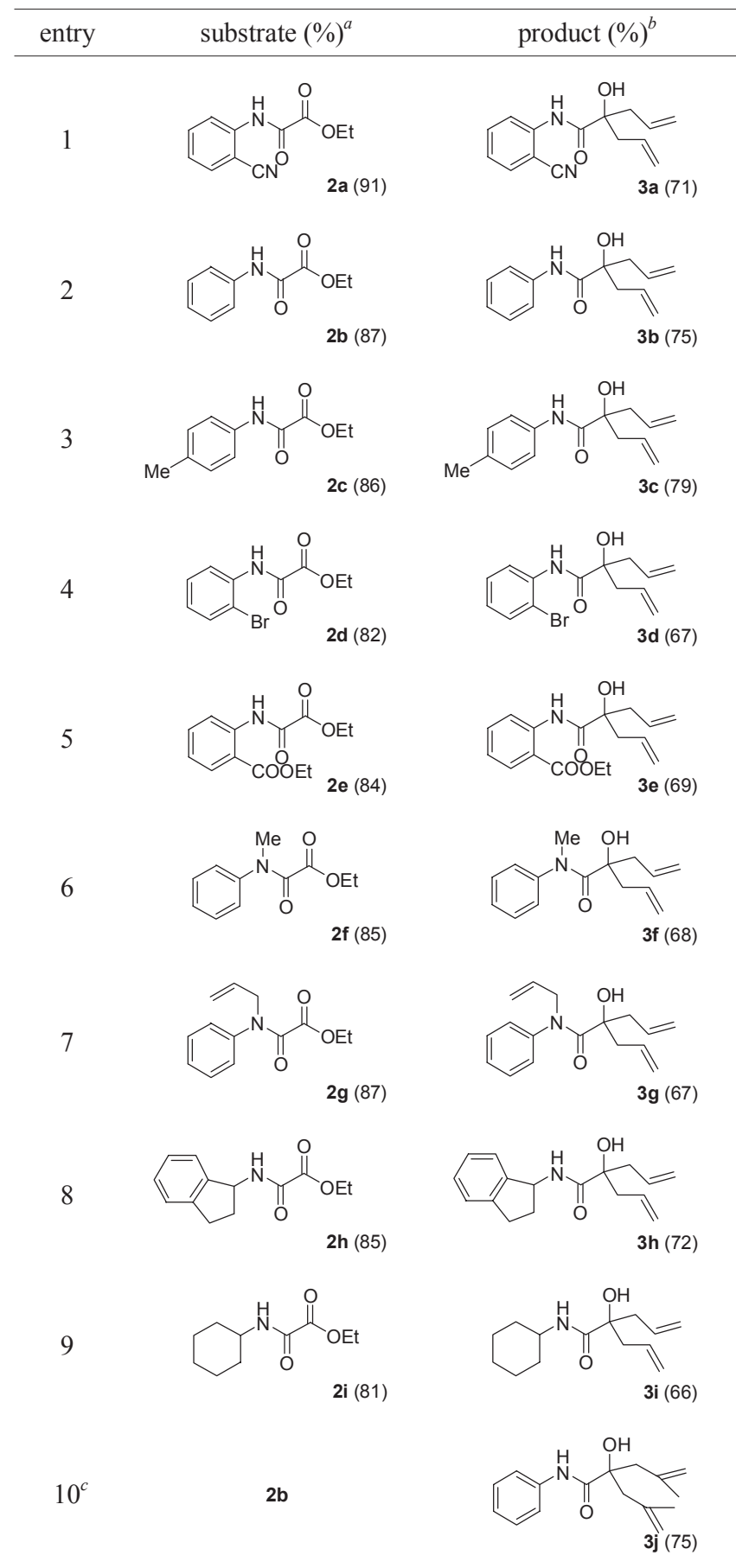

${ }^{a}$ Conditions: amine 1 (1.0 equiv), monoethyl oxalyl chloride (1.1 equiv), $\mathrm{Et}_{3} \mathrm{~N}$ (1.1 equiv), $\mathrm{CH}_{2} \mathrm{Cl}_{2}, 0^{\circ} \mathrm{C}(10 \mathrm{~min}), \mathrm{rt}(2 \mathrm{~h}) .{ }^{b}$ Conditions: amide-ester 2 ( 1.0 equiv), allyl bromide ( 4.0 equiv), In powder (2.0 equiv), THF, reflux, 5 min. ${ }^{c}$ Methallyl bromide was used. 
sence of an electron-withdrawing 2-pyridyl moiety in part. In addition, there could be a favorable chelation effect between the nitrogen atom and indium metal in the transition state. ${ }^{1}$ The reaction of $\alpha$-keto ester 8 produced 9 in moderate yield (60\%) at room temperature. Ketone group showed higher reactivity than the ester even though the ketone is sterically hindered. ${ }^{7}$ The reaction of diethyl oxalate (10) showed similar reactivity as that of oxalic amide-ester $\mathbf{2}$, and produced diallyl carbinol 11 in $58 \%$ yield.

In summary, we prepared various $\alpha, \alpha$-diallyl carbinols via the indium-mediated Barbier type allylation of amide-esters. From the results we found that allylindium reagents can react effectively with ester groups activated by amide or another ester group.

\section{Experimental Section}

Typical procedure for the synthesis of amide-ester $2 \mathrm{a}$. To a stirred solution of 2-aminobenzonitrile (1a, $118 \mathrm{mg}, 1.0 \mathrm{mmol}$ ) and $\mathrm{Et}_{3} \mathrm{~N}(111 \mathrm{mg}, 1.1 \mathrm{mmol})$ in $\mathrm{CH}_{2} \mathrm{Cl}_{2}(2 \mathrm{~mL})$ was added a solution of monoethyl oxalyl chloride $(150 \mathrm{mg}, 1.1 \mathrm{mmol}$, in $1 \mathrm{~mL}$ of $\left.\mathrm{CH}_{2} \mathrm{Cl}_{2}\right)$ at $0{ }^{\circ} \mathrm{C}(10 \mathrm{~min})$ and the reaction mixture was stirred at room temperature for $2 \mathrm{~h}$. After the usual aqueous workup and column chromatographic purification process (hexanes $\left./ \mathrm{CH}_{2} \mathrm{Cl}_{2} / \mathrm{EA}, 10: 1: 1\right)$, 2a was obtained as a white solid, $198 \mathrm{mg}(91 \%){ }^{4 \mathrm{~b}}$ Other compounds were prepared similarly as reported, ${ }^{4 \mathrm{a}-\mathrm{d}}$ and the spectroscopic data of unknown compounds $\mathbf{2 e}, \mathbf{2 g}$ and $\mathbf{2 h}$ are as follows.

Compound 2e: $84 \%$; white solid, mp $91-92{ }^{\circ} \mathrm{C}$; IR (KBr) $3242,1728,1687,1585,1516 \mathrm{~cm}^{-1} ;{ }^{1} \mathrm{HNMR}\left(\mathrm{CDCl}_{3}, 300 \mathrm{MHz}\right)$ $\delta 1.43(\mathrm{t}, J=7.2 \mathrm{~Hz}, 3 \mathrm{H}), 1.45(\mathrm{t}, J=7.2 \mathrm{~Hz}, 3 \mathrm{H}), 4.44$ (q, $J=$ $7.2 \mathrm{~Hz}, 2 \mathrm{H}), 4.45$ (q, $J=7.2 \mathrm{~Hz}, 2 \mathrm{H}), 7.19$ (t, $J=8.4 \mathrm{~Hz}, 1 \mathrm{H})$, $7.60(\mathrm{t}, J=8.1 \mathrm{~Hz}, 1 \mathrm{H}), 8.10(\mathrm{~d}, J=8.1 \mathrm{~Hz}, 1 \mathrm{H}), 8.75(\mathrm{~d}, J=$ $8.4 \mathrm{~Hz}, 1 \mathrm{H}), 12.63$ (br s, NH); ${ }^{13} \mathrm{C}$ NMR $\left(\mathrm{CDCl}_{3}, 75 \mathrm{MHz}\right) \delta$ $13.92,14.11,61.65,63.51,116.44,120.36,123.88,131.02$, 134.49, 139.50, 154.64, 160.57, 167.59; ESIMS m/z 266 $\left(\mathrm{M}^{+}+1\right)$.

Compound 2g: 87\%; colorless oil; IR (film) 1743, 1671, $1596,1495,1410 \mathrm{~cm}^{-1} ;{ }^{1} \mathrm{H} \mathrm{NMR}\left(\mathrm{CDCl}_{3}, 300 \mathrm{MHz}\right) \delta 0.98(\mathrm{t}$, $J=7.2 \mathrm{~Hz}, 3 \mathrm{H}), 4.01(\mathrm{q}, J=7.2 \mathrm{~Hz}, 2 \mathrm{H}), 4.37(\mathrm{~d}, J=6.3 \mathrm{~Hz}$, $2 \mathrm{H}), 5.15-5.18(\mathrm{~m}, 1 \mathrm{H}), 5.20-5.22(\mathrm{~m}, 1 \mathrm{H}), 5.80-5.92(\mathrm{~m}, 1 \mathrm{H})$, 7.21-7.26 (m, 2H), 7.33-7.38 (m, 3H); ${ }^{13} \mathrm{C} \mathrm{NMR}\left(\mathrm{CDCl}_{3}, 75\right.$ $\mathrm{MHz}) \delta 13.54,51.23,61.59,118.91,127.48,128.47,129.36$, 131.59, 140.00, 161.51, 162.47; ESIMS m/z $234\left(\mathrm{M}^{+}+1\right)$.

Compound 2h: 85\%; colorless oil; IR (film) 3354, 1691, $1681 \mathrm{~cm}^{-1} ;{ }^{1} \mathrm{H} \mathrm{NMR}\left(\mathrm{CDCl}_{3}, 300 \mathrm{MHz}\right) \delta 1.40(\mathrm{t}, J=7.2 \mathrm{~Hz}$, $3 \mathrm{H}), 1.85-1.97(\mathrm{~m}, 1 \mathrm{H}), 2.58-2.69(\mathrm{~m}, 1 \mathrm{H}), 2.85-3.09(\mathrm{~m}, 2 \mathrm{H})$, $4.35(\mathrm{q}, J=7.2 \mathrm{~Hz}, 2 \mathrm{H}), 5.50(\mathrm{dt}, J=7.8$ and $7.5 \mathrm{~Hz}, 1 \mathrm{H})$, 7.19-7.31 (m, $4 \mathrm{H}+\mathrm{NH}) ;{ }^{13} \mathrm{C} \mathrm{NMR}\left(\mathrm{CDCl}_{3}, 75 \mathrm{MHz}\right) \delta 13.96$, $30.23,35.51,55.08,63.26,124.14,124.90,126.90,128.36$, 141.78, 143.40, 156.26, 160.68; ESIMS m/z $234\left(\mathrm{M}^{+}+1\right)$.

Typical procedure for the synthesis of diallyl carbinol 3a. A stirred mixture of $2 \mathbf{a}(109 \mathrm{mg}, 0.5 \mathrm{mmol})$, allyl bromide (242 mg, $2.0 \mathrm{mmol})$, and indium powder $(114 \mathrm{mg}, 1.0 \mathrm{mmol})$ in THF $(0.5 \mathrm{~mL})$ was heated to reflux for $5 \mathrm{~min}$ under nitrogen atmosphere. After the usual aqueous workup and column chromatographic purification process (hexanes $\left./ \mathrm{CH}_{2} \mathrm{Cl}_{2} / \mathrm{EA}, 15: 2: 1\right)$, 3a was obtained as a white solid, $91 \mathrm{mg}(71 \%)$. Other compounds were prepared similarly and the spectroscopic data of unknown compounds $\mathbf{3 a - j} \mathbf{j}, \mathbf{7}$ and $\mathbf{9}$ are as follows.

Compound 3a: $71 \%$; white solid, mp $78-79{ }^{\circ} \mathrm{C}$; IR (KBr) $3316,2221,1679,1525 \mathrm{~cm}^{-1}$; ${ }^{1} \mathrm{H}$ NMR $\left(\mathrm{CDCl}_{3}, 300 \mathrm{MHz}\right) \delta$ 2.42-2.49 (m, 2H), 2.73-2.81 (m, 2H), $2.96(\mathrm{~s}, \mathrm{OH}), 5.19-5.26$ $(\mathrm{m}, 4 \mathrm{H}), 5.79-5.93(\mathrm{~m}, 2 \mathrm{H}), 7.16-7.22(\mathrm{~m}, 1 \mathrm{H}), 7.57-7.63(\mathrm{~m}$, $2 \mathrm{H}), 8.41(\mathrm{~d}, J=8.7 \mathrm{~Hz}, 1 \mathrm{H}), 9.31$ (br s, $\mathrm{NH}) ;{ }^{13} \mathrm{C} \mathrm{NMR}\left(\mathrm{CDCl}_{3}\right.$, $75 \mathrm{MHz}) \delta 43.14,77.50,102.50,116.02,120.50,120.77$, 124.25, 131.61, 132.26, 134.03, 139.74, 173.00; ESIMS m/z $257\left(\mathrm{M}^{+}+1\right)$. Anal. Calcd. For $\mathrm{C}_{15} \mathrm{H}_{16} \mathrm{~N}_{2} \mathrm{O}$ : C, 70.29; H, 6.29; N, 10.93. Found: C, 70.61; H, 6.45; N, 10.59 .

Compound 3b: $75 \%$; white solid, mp $38-39{ }^{\circ} \mathrm{C}$; IR (KBr) $3369,1664,1529,1445 \mathrm{~cm}^{-1} ;{ }^{1} \mathrm{H}$ NMR $\left(\mathrm{CDCl}_{3}, 300 \mathrm{MHz}\right) \delta$ $2.36-2.43(\mathrm{~m}, 2 \mathrm{H}), 2.68(\mathrm{~s}, \mathrm{OH}), 2.77-2.83(\mathrm{~m}, 2 \mathrm{H}), 5.18-5.23$ $(\mathrm{m}, 4 \mathrm{H}), 5.76-5.90(\mathrm{~m}, 2 \mathrm{H}), 7.12(\mathrm{t}, J=8.4 \mathrm{~Hz}, 1 \mathrm{H}), 7.33(\mathrm{t}, J=$ $8.4 \mathrm{~Hz}, 2 \mathrm{H}), 7.56(\mathrm{~d}, J=8.4 \mathrm{~Hz}, 2 \mathrm{H}), 8.60(\mathrm{br} \mathrm{s}, \mathrm{NH}) ;{ }^{13} \mathrm{C} \mathrm{NMR}$ $\left(\mathrm{CDCl}_{3}, 75 \mathrm{MHz}\right) \delta 43.29,77.04,119.66,120.29,124.42$, 128.96, 132.15, 137.17, 172.23; ESIMS m/z $232\left(\mathrm{M}^{+}+1\right)$. Anal. Calcd. For $\mathrm{C}_{14} \mathrm{H}_{17} \mathrm{NO}_{2}$ : C, 72.70; H, 7.41; N, 6.06. Found: C, 72.87; H, 7.66; N, 5.89 .

Compound 3c: $79 \%$; white solid, mp $50-51{ }^{\circ} \mathrm{C}$; IR (KBr) $3373,1660,1593,1523 \mathrm{~cm}^{-1} ;{ }^{1} \mathrm{H}$ NMR $\left(\mathrm{CDCl}_{3}, 300 \mathrm{MHz}\right) \delta$ $2.31(\mathrm{~s}, 3 \mathrm{H}), 2.35-2.42(\mathrm{~m}, 2 \mathrm{H}), 2.58(\mathrm{~s}, \mathrm{OH}), 2.76-2.83(\mathrm{~m}$, $2 \mathrm{H}), 5.18-5.23(\mathrm{~m}, 4 \mathrm{H}), 5.76-5.90(\mathrm{~m}, 2 \mathrm{H}), 7.13(\mathrm{~d}, J=8.4 \mathrm{~Hz}$, $2 \mathrm{H}), 7.44$ (d, $J=8.4 \mathrm{~Hz}, 2 \mathrm{H}), 8.51$ (br s, $\mathrm{NH}) ;{ }^{13} \mathrm{C} \mathrm{NMR}\left(\mathrm{CDCl}_{3}\right.$, $75 \mathrm{MHz}) \delta 20.84,43.31,77.00,119.70,120.23,129.45,132.24$, 134.05, 134.67, 172.04; ESIMS m/z $246\left(\mathrm{M}^{+}+1\right)$.

Compound 3d: $67 \%$; white solid, mp $72-73{ }^{\circ} \mathrm{C}$; IR (KBr) $3424,3342,1672,1524,1438 \mathrm{~cm}^{-1}$; ${ }^{1} \mathrm{HNMR}\left(\mathrm{CDCl}_{3}, 300 \mathrm{MHz}\right)$ $\delta 2.39-2.46(\mathrm{~m}, 2 \mathrm{H}), 2.76-2.83(\mathrm{~m}, 2 \mathrm{H}), 2.77(\mathrm{~s}, \mathrm{OH}), 5.18-5.25$ $(\mathrm{m}, 4 \mathrm{H}), 5.78-5.92(\mathrm{~m}, 2 \mathrm{H}), 6.98(\mathrm{t}, J=8.1 \mathrm{~Hz}, 1 \mathrm{H}), 7.31(\mathrm{t}, J=$ $8.1 \mathrm{~Hz}, 1 \mathrm{H}), 7.54(\mathrm{~d}, J=8.1 \mathrm{~Hz}, 1 \mathrm{H}), 8.37(\mathrm{~d}, J=8.1 \mathrm{~Hz}, 1 \mathrm{H})$, 9.19 (br s, NH); ${ }^{13} \mathrm{C} \mathrm{NMR}\left(\mathrm{CDCl}_{3}, 75 \mathrm{MHz}\right) \delta 43.25,77.40$, 113.83, 120.33, 121.47, 125.28, 128.22, 131.95, 132.26, 135.22, 172.55; ESIMS $m / z 310\left(\mathrm{M}^{+}+1\right), 312\left(\mathrm{M}^{+}+3\right)$.

Compound 3e: 69\%; white solid, mp 93 - $94{ }^{\circ} \mathrm{C}$; IR (KBr) $3297,3239,1703,1661,1584 \mathrm{~cm}^{-1} ;{ }^{1} \mathrm{HNMR}\left(\mathrm{CDCl}_{3}, 300 \mathrm{MHz}\right)$ $\delta 1.45(\mathrm{t}, J=6.9 \mathrm{~Hz}, 3 \mathrm{H}), 2.46-2.53(\mathrm{~m}, 2 \mathrm{H}), 2.76-2.84(\mathrm{~m}$, $2 \mathrm{H}), 2.91(\mathrm{~s}, \mathrm{OH}), 4.44(\mathrm{q}, J=6.9 \mathrm{~Hz}, 2 \mathrm{H}), 5.18-5.26(\mathrm{~m}, 4 \mathrm{H})$, $5.81-5.95(\mathrm{~m}, 2 \mathrm{H}), 7.15(\mathrm{t}, J=7.8 \mathrm{~Hz}, 1 \mathrm{H}), 7.58(\mathrm{t}, J=7.8 \mathrm{~Hz}$, $1 \mathrm{H}), 8.11(\mathrm{~d}, J=7.8 \mathrm{~Hz}, 1 \mathrm{H}), 8.79(\mathrm{~d}, J=7.8 \mathrm{~Hz}, 1 \mathrm{H}), 11.91$ (br s, NH); ${ }^{13} \mathrm{C} \mathrm{NMR}\left(\mathrm{CDCl}_{3}, 75 \mathrm{MHz}\right) \delta 14.21,43.43,61.39$, 77.31, 116.04, 119.94, 120.21, 122.80, 130.94, 132.24, 134.38, 140.60, 167.80, 173.69; ESIMS m/z $304\left(\mathrm{M}^{+}+1\right)$. Anal. Calcd. For $\mathrm{C}_{17} \mathrm{H}_{21} \mathrm{NO}_{4}$ : C, 67.31; H, 6.98; N, 4.62. Found: C, 67.43; $\mathrm{H}, 7.08 ; \mathrm{N}, 4.44$.

Compound 3f: 68\%; colorless oil; IR (film) 3395, 1630, 1594, $1356 \mathrm{~cm}^{-1} ;{ }^{1} \mathrm{H} \mathrm{NMR}\left(\mathrm{CDCl}_{3}, 300 \mathrm{MHz}\right) \delta 2.25$ (br s, 4H), 3.31 $(\mathrm{s}, 3 \mathrm{H}), 4.30$ (br s, OH), 5.05-5.16 (m, 4H), 5.76-5.89 (m, 2H), 7.25-7.28 (m, 2H), 7.33-7.45 (m, 3H); ${ }^{13} \mathrm{C} \mathrm{NMR}\left(\mathrm{CDCl}_{3}, 75\right.$ $\mathrm{MHz}) \delta 41.07,43.51,77.36,118.24,127.86,128.47,129.30$, 132.90, 143.58, 174.03; ESIMS $m / z 246\left(\mathrm{M}^{+}+1\right)$.

Compound 3g: 67\%; colorless oil; IR (film) 3403, 1628, $1594,1369 \mathrm{~cm}^{-1}$; ${ }^{1} \mathrm{H}$ NMR $\left(\mathrm{CDCl}_{3}, 300 \mathrm{MHz}\right) \delta 2.20(\mathrm{~d}, J=$ $6.0 \mathrm{~Hz}, 4 \mathrm{H}), 4.26(\mathrm{~d}, J=8.0 \mathrm{~Hz}, 2 \mathrm{H}), 4.27$ (s, OH), 4.96-5.16 $(\mathrm{m}, 6 \mathrm{H}), 5.75-5.93(\mathrm{~m}, 3 \mathrm{H}), 7.23-7.27(\mathrm{~m}, 2 \mathrm{H}), 7.37-7.42(\mathrm{~m}$, $3 \mathrm{H}) ;{ }^{13} \mathrm{C} \mathrm{NMR}\left(\mathrm{CDCl}_{3}, 75 \mathrm{MHz}\right) \delta 43.52,56.10,77.31,118.31$, 118.54, 128.71, 129.00 (2C), 132.36, 132.86, 141.74, 173.54; ESIMS $m / z 272\left(\mathrm{M}^{+}+1\right)$. 
Compound 3h: 72\%; colorless oil; IR (film) 3393, 3314, $1650,1524 \mathrm{~cm}^{-1} ;{ }^{1} \mathrm{H} \mathrm{NMR}\left(\mathrm{CDCl}_{3}, 300 \mathrm{MHz}\right) \delta 1.72-1.86(\mathrm{~m}$, $1 \mathrm{H}), 2.31-2.40(\mathrm{~m}, 2 \mathrm{H}), 2.52-2.61(\mathrm{~m}, 1 \mathrm{H}), 2.62(\mathrm{~s}, \mathrm{OH}), 2.67-$ $2.77(\mathrm{~m}, 2 \mathrm{H}), 2.80-3.05(\mathrm{~m}, 2 \mathrm{H}), 5.14-5.23(\mathrm{~m}, 4 \mathrm{H}), 5.43(\mathrm{dt}$, $J=8.1$ and $7.8 \mathrm{~Hz}, 1 \mathrm{H}), 5.73-5.92(\mathrm{~m}, 2 \mathrm{H}), 6.93(\mathrm{~d}, J=8.1 \mathrm{~Hz}$, $\mathrm{NH}), 7.16-7.29(\mathrm{~m}, 4 \mathrm{H}) ;{ }^{13} \mathrm{C} \mathrm{NMR}\left(\mathrm{CDCl}_{3}, 75 \mathrm{MHz}\right) \delta 30.19$, 34.00, 43.36, 43.42, 54.34, 76.64, 119.74, 119.86, 123.99, 124.73, 126.68, 127.88, 132.39, 132.47, 142.98, 143.32, 173.69; ESIMS $m / z 272\left(\mathrm{M}^{+}+1\right)$.

Compound 3i: 66\%; colorless oil; IR (film) 3396, 3334, $1646,1530 \mathrm{~cm}^{-1}$; ${ }^{1} \mathrm{H} \mathrm{NMR}\left(\mathrm{CDCl}_{3}, 300 \mathrm{MHz}\right) \delta 1.10-1.23(\mathrm{~m}$, $3 \mathrm{H}), 1.28-1.43$ (m, 2H), 1.58-1.74 (m, 3H), 1.84-1.89 (m, 2H), 2.27-2.35 (m, 2H), 2.64-2.72 (m, 2H), $2.72(\mathrm{~s}, \mathrm{OH}), 3.67-3.80$ $(\mathrm{m}, 1 \mathrm{H}), 5.12-5.21(\mathrm{~m}, 4 \mathrm{H}), 5.71-5.84(\mathrm{~m}, 2 \mathrm{H}), 6.62(\mathrm{~d}, J=7.8$ $\mathrm{Hz}, \mathrm{NH}) ;{ }^{13} \mathrm{C} \mathrm{NMR}\left(\mathrm{CDCl}_{3}, 75 \mathrm{MHz}\right) \delta 27.71,25.42,33.04$, 43.33, 47.90, 76.34, 119.48, 132.50, 172.85; ESIMS m/z 238 $\left(\mathrm{M}^{+}+1\right)$. Anal. Calcd. For $\mathrm{C}_{14} \mathrm{H}_{23} \mathrm{NO}_{2}: \mathrm{C}, 70.85 ; \mathrm{H}, 9.77 ; \mathrm{N}$, 5.90. Found: C, 70.96; H, 9.65; N, 5.77.

Compound 3j: $75 \%$; white solid, mp $75-77{ }^{\circ} \mathrm{C}$; IR (KBr) $3438,3373,1665,1600,1527,1444 \mathrm{~cm}^{-1} ;{ }^{1} \mathrm{H} \mathrm{NMR}\left(\mathrm{CDCl}_{3}\right.$, $300 \mathrm{MHz}) \delta 1.77(\mathrm{~s}, 6 \mathrm{H}), 2.34(\mathrm{~d}, J=13.5 \mathrm{~Hz}, 2 \mathrm{H}), 2.83(\mathrm{~s}$, $\mathrm{OH}), 2.86(\mathrm{~d}, J=13.5 \mathrm{~Hz}, 2 \mathrm{H}), 4.86(\mathrm{~s}, 2 \mathrm{H}), 4.96(\mathrm{~s}, 2 \mathrm{H}), 7.11$ $(\mathrm{t}, J=7.5 \mathrm{~Hz}, 1 \mathrm{H}), 7.32(\mathrm{t}, J=8.1 \mathrm{~Hz}, 2 \mathrm{H}), 7.55(\mathrm{~d}, J=8.4 \mathrm{~Hz}$, $2 \mathrm{H}), 8.72$ (br s, NH); ${ }^{13} \mathrm{C} \mathrm{NMR}\left(\mathrm{CDCl}_{3}, 75 \mathrm{MHz}\right) \delta 23.68$, 47.19, 76.40, 116.33, 119.65, 124.36, 128.98, 137.40, 141.39, 172.71; ESIMS m/z $260\left(\mathrm{M}^{+}+1\right)$. Anal. Calcd. For $\mathrm{C}_{16} \mathrm{H}_{21} \mathrm{NO}_{2}$ : C, 74.10; H, 8.16; N, 5.40. Found: C, 74.42; H, 8.36; N, 5.13.

Compound 7: 52\%; colorless oil; IR (film) 3391, 1640, 1592, $1435,1393 \mathrm{~cm}^{-1}$; ${ }^{1} \mathrm{H}$ NMR $\left(\mathrm{CDCl}_{3}, 300 \mathrm{MHz}\right) \delta 2.62$ (dt, $J=7.2$ and $1.2 \mathrm{~Hz}, 4 \mathrm{H}), 4.91(\mathrm{~s}, \mathrm{OH}), 4.96-5.03(\mathrm{~m}, 4 \mathrm{H}), 5.58-5.72$ (m, 2H), 7.18 (ddd, $J=12.3,5.1$ and $1.2 \mathrm{~Hz}, 1 \mathrm{H}), 7.33(\mathrm{dt}, J=$ 8.1 and $1.2 \mathrm{~Hz}, 1 \mathrm{H}), 7.69(\mathrm{td}, J=8.1$ and $1.8 \mathrm{~Hz}, 1 \mathrm{H}), 8.51-8.54$ $(\mathrm{m}, 1 \mathrm{H}),{ }^{13} \mathrm{C} \mathrm{NMR}\left(\mathrm{CDCl}_{3}, 75 \mathrm{MHz}\right) \delta 46.08,75.54,118.15$, $120.03,121.81,133.50,136.56,147.49,162.85 ;$ ESIMS $m / z$ $190\left(\mathrm{M}^{+}+1\right)$. Anal. Calcd. For $\mathrm{C}_{12} \mathrm{H}_{15} \mathrm{NO}$ : C, 76.16; H, 7.99; N, 7.40. Found: C, 76.23; H, 8.16; N, 7.29.

Compound 9: 60\%; colorless oil; IR (film) 3485, $1729 \mathrm{~cm}^{-1}$; ${ }^{1} \mathrm{H} \mathrm{NMR}\left(\mathrm{CDCl}_{3}, 300 \mathrm{MHz}\right) \delta 1.26(\mathrm{t}, J=7.2 \mathrm{~Hz}, 3 \mathrm{H}), 2.23(\mathrm{~s}$, $3 \mathrm{H}), 2.37$ (s, 6H), 2.84-3.03 (m, 2H), $2.88(\mathrm{~s}, \mathrm{OH}), 4.22(\mathrm{q}, J=$ $7.2 \mathrm{~Hz}, 2 \mathrm{H}), 5.11-5.18(\mathrm{~m}, 2 \mathrm{H}), 5.80-5.94(\mathrm{~m}, 1 \mathrm{H}) 6.80(\mathrm{~s}$, $2 \mathrm{H}) ;{ }^{13} \mathrm{C} \mathrm{NMR}\left(\mathrm{CDCl}_{3}, 75 \mathrm{MHz}\right) \delta 13.89,20.41,22.87,42.45$, 61.60, 80.49, 119.17, 131.55, 133.07, 135.25, 136.12, 136.44, 174.14; ESIMS m/z $263\left(\mathrm{M}^{+}+1\right)$. Anal. Calcd. For $\mathrm{C}_{16} \mathrm{H}_{22} \mathrm{O}_{3}$ : C, 73.25; H, 8.45. Found: C, 73.44; H, 8.29.

Acknowledgments. This research was supported by Basic Science Research Program through the National Research Foundation of Korea (NRF) funded by the Ministry of Education, Science and Technology (2009-0070633). Spectroscopic data was obtained from the Korea Basic Science Institute, Gwangju branch.

\section{References and Notes}

1. For the general review on indium-mediated reactions, see: (a) Auge, J.; Lubin-Germain, N.; Uziel, J. Synthesis 2007, 1739-1764. (b) Kargbo, R. B.; Cook, G. R. Curr. Org. Chem. 2007, 11, 12871309. (c) Lee, P. H. Bull. Korean Chem. Soc. 2007, 28, 17-28. (d) Li, C.-J.; Chan, T.-H. Tetrahedron 1999, 55, 11149-11176. (e) Pae, A. N.; Cho, Y. S. Curr. Org. Chem. 2002, 6, 715-737. (f) Nair, V.; Ros, S.; Jayan, C. N.; Pillai, B. S. Tetrahedron 2004, 60, 19591982. (g) Podlech, J.; Maier, T. C. Synthesis 2003, 633-655.

2. (a) Fujiwara, N.; Yamamoto, Y. Tetrahedron Lett. 1998, 39, 47294732. (b) Fujiwara, N.; Yamamoto, Y. J. Org. Chem. 1999, 64, 4095-4101. For diallylation of benzonitrile with allylindate, see: (c) Jin, S.-J.; Araki, S.; Butsugan, Y. Bull. Chem. Soc. Jpn. 1993, $66,1528-1532$. For the indium(I) iodide-promoted allylation of $\alpha, \beta$-unsaturated nitrile, see: (d) Ranu, B. C.; Das, A. Tetrahedron Lett. 2004, 45, 6875-6877.

3. For the In-mediated Barbier type allylation of nitrile-containing substrates, see: (a) Kim, S. H.; Lee, H. S.; Kim, K. H.; Kim, J. N. Tetrahedron Lett. 2009, 50, 1696-1698. (b) Kim, S. H.; Kim, S. H.; Lee, K. Y.; Kim, J. N. Tetrahedron Lett. 2009, 50, 5744-5747. (c) Kim, S. H.; Lee, H. S.; Kim, K. H.; Kim, J. N. Tetrahedron Lett. 2009, 50, 6476-6479. (d) Kim, S. H. Kim, S. H.; Kim, K. H.; Kim, J. N. Tetrahedron Lett. 2010, 51, 860-862. (e) Kim, S. H.; Kim, S. H.; Kim, T. H.; Kim, J. N. Tetrahedron Lett. 2010, 51, 2774-2777.

4. For the synthesis of starting materials, see: (a) Langer, P.; Schroeder, R. Eur. J. Org. Chem. 2004, 1025-1032. (b) Chakraborty, K.; Devakumar, C.; Tomar, S. M. S.; Kumar, R. J. Agric. Food Chem. 2003, 51, 992-998. (c) Sellstedt, J. H.; Guinosso, C. J.; Begany, A. J.; Bell, S. C.; Rosenthale, M. J. Med. Chem. 1975, 18, 926-933. (d) Kuhn, C.; Beckert, R.; Friedrich, M.; Gorls, H. J. Heterocyclic Chem. 2006, 43, 1569-1574.

5. For the synthesis and synthetic applications of diallyl carbinols, see: (a) Kitagawa, O.; Momose, S.-i.; Fushimi, Y.; Taguchi, T. Tetrahedron Lett. 1999, 40, 8827-8831. (b) Cao, H.; Mundla, S. R.; Cook, J. M. Tetrahedron Lett. 2003, 44, 6165-6168. (c) Van Ornum, S. G.; Bruendl, M. M.; Cao, H.; Reddy, M.; Grubisha, D. S.; Bennett, D. W.; Cook, J. M. J. Org. Chem. 2000, 65, 1957-1971. (d) Macritchie, J. A.; Silcock, A.; Willis, C. L. Tetrahedron: Asymmetry 1997, 8, 3895-3902. (e) Van Ornum, S. G.; Cook, J. M. Tetrahedron Lett. 1996, 37, 7185-7188. (f) Kitagawa, O.; Hanano, T.; Tanabe, K.; Shiro, M.; Taguchi, T. J. Chem. Soc., Chem. Commun. 1992, 1005-1007. (g) Jia, Y.; Zhang, M.; Tao, F.; Zhou, J. Synth. Commun. 2002, 32, 2829-2835. (h) Schmidt, B.; Pohler, M. Org. Biomol. Chem. 2003, 1, 2512-2517.

6. For the reactions of allylindium reagents and cyclic amide, $N$-acyl pyrazole, $N$-acyl imidazole, and 2-pyridyl carboxylates, see: (a) Coleman, R. S.; Walczak, M. C.; Campbell, E. L. J. Am. Chem. Soc. 2005, 127, 16038-16039. (b) Bryan, V. J.; Chan, T.-H. Tetrahedron Lett. 1997, 38, 6493-6496. (c) Yoo, J.; Oh, K. E.; Keum, G.; Kang, S. B.; Kim, Y. Polyhedron 2000, 19, 549-551. For the Barbier type allylation of ester in the presence of aluminum metal and lead(II) bromide, see: (d) Tanaka, H.; Nakahata, S.; Watanabe, H.; Zhao, J.; Kuroboshi, M.; Torii, S. Inorg. Chim. Acta 1999, 296, 204-207.

7. For the selective allylation of ketone in the presence of ester, acid, and related functional groups, see: (a) Lee, P. H.; Seomoon, D.; Lee, K. Bull. Korean Chem. Soc. 2001, 22, 1380-1384. (b) Li, C.-J.; Lu, Y.-Q. Tetrahedron Lett. 1995, 36, 2721-2724. (c) Lee, P. H.; Lee, K.; Chang, S. Synth. Commun. 2001, 31, 3189-3196. (d) Kaur, P.; Singh, P.; Kumar, S. Tetrahedron 2005, 61, 8231-8240. 